\title{
On-line charge breeding using ECRIS and EBIS
}

\author{
Richard Vondrasek \\ Argonne National Laboratory, Physics Division, Lemont, Illinois, 60439 United States of America \\ e-mail: Vondrasek@anl.gov
}

\begin{abstract}
The efficient and rapid production of a high-quality, pure beam of highly charged ions is at the heart of any radioactive ion beam facility. Whether an electron cyclotron resonance (ECR) ion source or an electron beam ion source (EBIS) is used to produce these highly charged ions, their operating characteristics will set the boundaries on the range of experiments which can be performed. In addition, time structure and duty cycle have to be considered when defining the operating parameters of the accelerator system as a whole. At Argonne National Laboratory (ANL), an ECR charge breeder was developed as part of the Californium Rare Ion Breeder Upgrade (CARIBU) program. The charge breeding efficiency and high charge state production of the source is at the forefront of ECR charge breeders, but its overall performance as part of the accelerator system is limited by pervasive background and relatively long breeding times. As such, an EBIS charge breeder has been developed and is running in an off-line configuration. It has already demonstrated good breeding efficiencies, shorter residence times, and reduced background and is scheduled to replace the ECR charge breeder in late 2015. The resultant change in duty cycle and time structure necessitates changes to the overall operation of the facility. The experiences with these breeders, as well as from several other facilities which already utilize an ECR or EBIS for charge breeding, help to define the operational characteristics of each technology - their strengths, their weaknesses, and the possible paths to improvement.
\end{abstract}

Keywords: Charge breeding, Radioactive ion beam, Electron cyclotron resonance ion source, Electron beam ion source, Background contamination

\section{Introduction}

As more charge breeders come on line, the variety of design and the operational experience gained are helping to better define solutions to the problems encountered with the charge breeding technique. New breeders are being commissioned for SPES [1], SPIRAL [2], and VECC [3] with others in the planning and design stages [4]. Faced with many of the same operational challenges - predominantly beam purity for ECR breeders and for EBIS breeders the efficient injection of a large number of particles accompanied by a slow extraction - these new breeders incorporate into their design and construction many of the lessons learned from existing charge breeders, and as such, the performance gap between the two devices is narrowing.

\section{ECR charge breeding}

ECR sources have been utilized as charge breeders for many years, first to ionize radioactive species which were introduced into the source via a carrier gas [5] and later to ionize radioactive species introduced directly into the plasma as 1+ ions [6]. A room temperature ECR ion source has solenoid coils providing an axial confining field and a permanent magnet hexapole providing radial confinement. The plasma is excited by RF typically in the $10-14 \mathrm{GHz}$ range. For charge breeding, the 1+ ions are introduced into the plasma from the injection side of the source, thermalized in the plasma, undergo stepwise ionization via collisions with energetic electrons, and are then extracted for subsequent acceleration [7]. It is a CW device which can accept several e $\mu \mathrm{A}$ of injected beam, has a good efficiency, and can produce the high-quality, highly-charged ion beam necessary for injection into an accelerator system [8].

Figure 1 shows the charge breeding performance for the Phoenix ECR sources tested at ISOLDE [9] and LPSC [10], the TRIAC source [11], the TRIUMF source [12], the ANL source [13, 14], and the recently commissioned SPES 
charge breeder (built by LPSC for the LNL group) [15]. The radioactive beam species are denoted with a halo around them.

\section{EBIS charge breeding}

The EBIS fulfills the same role as the ECR, the production of highly charged ions, but its confining fields are produced via trap electrodes, a superconducting solenoid, and the electron beam itself. Ionization is still stepwise, but in contrast to the broad electron energy distribution produced in an ECR stretching from a few $\mathrm{eV}$ to an $\mathrm{MeV}$ [16], the EBIS electron beam is monoenergetic allowing greater control over the peak charge state produced and the time it takes for its production. For charge breeding, the 1+ ions enter the trap region, an electrostatic barrier is then raised. Provided there is good overlap with the electron beam, the ions undergo stepwise ionization and after some time the trap is opened releasing the highly-charged ions [17]. An EBIS is an inherently pulsed device and requires preparation of the 1+ beam in a RFQ cooler/buncher before charge breeding in order to attain the highest efficiency [18].

Up until recently, the only data for EBIS charge breeding has been from the REX-EBIS group [19] shown in Figure 2. Over the last decade, their EBIS has demonstrated efficiencies as high as $23 \%$, and this includes the efficiency of the REXTRAP which prepares the ions for injection into the EBIS. Two new data points have been added to this graph - a potassium beam produced by the MSU ReA EBIT in 2013 [20] and a cesium beam recently produced by the EBIS at Argonne [21].

\section{ANL charge breeding program}

In support of CARIBU - a program to provide radioactive species for the ATLAS experimental program [22] - ANL has developed an ECR breeder as well as an EBIS breeder. The ECR breeder has been delivering charge bred radioactive ion beams to the ATLAS experimental program for the last several years. While its charge breeding efficiency and high charge state production have been at the forefront of ECR charge breeding, its overall performance as a part of the accelerator system has been hindered by the pervasive background present in ECR ion sources.

As such, the EBIS breeder has recently been commissioned and is scheduled to replace the ECR in late 2015. The primary motivation for replacing the ECR with an EBIS is the level of beam contamination present in an ECR typically on the order of several pA. Additional benefits of an EBIS include an improved charge breeding efficiency and faster breeding time $[23,24]$. However, the fact that an EBIS is a pulsed device as opposed to the CW nature of the ECR necessitates changes in accelerator operation and the manner in which beam will be delivered to target.

\subsection{ANL ECR charge breeder}

The ANL ECR breeder [25] (Figure 3) is a room temperature source, and the plasma is excited with two RF frequencies - a $10.44 \mathrm{GHz}$ klystron and an $11-13 \mathrm{GHz}$ traveling wave tube amplifier (TWTA). It has an open hexapole structure providing good pumping to the plasma chamber region resulting in a base plasma chamber pressure of $2 \times 10^{-8}$ Torr. The open structure also allows the RF and support gas to be introduced into the plasma chamber between the hexapole bars. This scheme eliminates the need for cut-outs in the field shaping iron to accept the RF waveguides and results in a highly symmetric axial magnetic field where the ions enter the plasma. This differs from other ECR breeders presently in existence which are closed hexapole devices with the RF injected along the long axis of the source. The 1+ ions are introduced into the plasma through a grounded high-purity aluminum tube mounted on a linear motion stage. The stage has a $30 \mathrm{~mm}$ range of travel, and thus the deceleration point of the $1+$ ions can be adjusted on-line without disturbing the source conditions. The source is designed to operate at a 50 $\mathrm{kV}$ potential although it typically operates at $36 \mathrm{kV}$.

To set up the charge breeder and develop charge breeding techniques, stable 1+ beams are produced by a surface ionization source or an RF discharge source with injected intensities ranging from 2-500 enA. The 1+ diagnostics station includes a fully shielded Faraday cup for measuring the stable 1+ beam (FC1) and an aluminum foil shrouded silicon surface barrier detector (SBD1) for measuring radioactive beams via beta decay. The $\mathrm{n}+$ diagnostics station includes a Faraday cup (FC2) for the stable beams and a silicon barrier detector (identical to SBD1) for the radioactive beams. The breeding efficiency and time are determined by using an electrostatic steerer 
to pulse the incoming $1+$ beam and measuring the $\mathrm{n}+$ response. The charge breeding time is defined as the time between injection of the $1+$ beam and the $n+$ beam reaching $90 \%$ of the maximum current (a typical trace for $\mathrm{Xe}^{29+}$ is shown in Figure 4).

\subsection{Effect of RF frequency on charge breeding efficiency and time}

The charge breeding time is more accurately the total time it takes to both create the ion and extract it from the plasma, and the breeding time as well as efficiency can vary significantly based upon the RF power levels and operating frequencies. The change in breeding efficiency and time was investigated using ${ }^{132} \mathrm{Xe}$ for two cases: one with the TWTA operating at $11.762 \mathrm{GHz}$ and the other operating at $11.765 \mathrm{GHz}$, both at 300W RF power. The ECR breeder was run with an oxygen plasma and single frequency heating employing only the TWTA. When the TWTA frequency was shifted, several effects were observed: the charge state distribution moved to higher charge states, the breeding efficiencies for the high charge state ions increased, and the breeding times increased. No other source parameters were changed between these two data sets implying that the frequency shift was the sole cause of the change in the plasma properties, possibly due to a change in the plasma potential as observed in [26]. Table 1 summarizes the observed charge breeding efficiencies and times. Clearly the choice of operating frequency is just as critical with charge breeding as has been demonstrated in normal ECR operation [27]. While the exact mechanism of the operating frequency effect is still being investigated, it does give the source operator another convenient knob with which to optimize the ion source performance.

While the highest breeding efficiency is always desired, achieving it may result in a long breeding time. For species such as ${ }^{139} \mathrm{Xe}$ with a $39.7 \mathrm{sec}$ half-life, a $2 \mathrm{sec}$ breeding time is not problematic. However, for a shorter lived species such as ${ }^{143} \mathrm{Xe}$ with a $0.3 \mathrm{sec}$ half-life, a short breeding time is required and this may result in a lower than optimum breeding efficiency. A compromise between ions lost to in-source decay and ions lost to a lower breeding efficiency can be established in order to optimize beam production.

\subsection{Background contamination}

The background contamination which plagues ECR charge breeders has been well documented [11, 28, 29]. A detailed mass scan of an oxygen plasma in the A/q region in which the ANL ECR breeder operates reveals that in addition to oxygen, we see constituents of nitrogen, argon, aluminum, fluorine, and chlorine on the $>1$ epA scale. There are still clean A/q regions within the spectrum that show no background as measured with a picoammeter, and these regions correspond with low rates observed with a silicon barrier detector (taken after acceleration in the linac). For example, ${ }^{143} \mathrm{Cs}^{27+}$ which is very close to the ${ }^{16} \mathrm{O}^{3+}$ peak has a $330 \mathrm{kHz}$ background rate, whereas ${ }^{146} \mathrm{Ba}^{28+}$ which is in a clean $\mathrm{A} / \mathrm{q}$ region has only $500 \mathrm{~Hz}$ background.

But even in the regions defined as 'clean', once the beam is accelerated to higher energies and can be observed with a silicon barrier detector, a number of stable background components are observed, as shown in Figure 5. In the case of ${ }^{146} \mathrm{Ba}^{28+}$, there are not as many contaminants as the conflict table predicts $(7$ contaminants were observed out of a possible 47), but the stable beam still accounted for $97 \%$ of the rate into the detector. Due to there being no strong contaminant near mass 146 , the ${ }^{146} \mathrm{Ba}^{28+}$ radioactive beam component was still easily identified. The observed contaminants of titanium, iron, chromium, zinc, molybdenum, tin, and mercury were routinely seen in other spectra and provided an indication of the material source.

\subsection{Sources of contamination}

There are three sources of contamination - gases entering the system via leaks or the support gas, loose particulates on the vacuum and beamline chamber surfaces, and contamination of the bulk plasma chamber material.

Investigation with a residual gas analyzer (RGA) showed that the nitrogen and argon contaminants were not constituents of the oxygen support gas. They were mainly due to o-ring permeation and to a smaller extent desorption from the plasma chamber and beamline surfaces. The o-rings are integral to the source construction and cannot be eliminated, and their presence results in the equivalent of a $10^{-5}$ torr-1/sec leak limiting us to an ultimate pressure of $2 \times 10^{-8}$ Torr. In order to decrease the level of wall desorption, a standard thermal bake-out technique would ordinarily be employed, but the presence of the permanent magnet hexapole and the high voltage isolation materials which are all susceptible to heat damage precluded this. The plasma chamber was instead baked out with 
UV lamps, and while the base pressure was reduced by a factor of 2 mainly due to decreased water desorption, there was only a small effect on the overall contaminant load.

In order to reduce contamination due to particulates, the KEK charge breeding group utilized sand blasting and high pressure rinsing of their plasma chamber [11]. In the case of the ANL charge breeder, it was not practical to disassemble the ion source so alternative cleaning methods were investigated. It was decided to utilize $\mathrm{CO}_{2}$ snow cleaning of the plasma chamber surfaces as well as the injection and extraction hardware. The method is nondestructive, nonabrasive, and residue-free. It is based upon the expansion of either liquid or gaseous carbon dioxide through an orifice leading to the nucleation of small dry ice particles in a high velocity gas carrier stream. The $\mathrm{CO}_{2}$ pellets remove micron and submicron particulates by momentum transfer and hydrocarbons via a freezefracture mechanism. The high-velocity carrier gas propels the contaminants out of the system thus eliminating the need for high pressure rinsing and allowing the entire process to be done in situ.

The last source of contamination is the plasma chamber itself which is constructed of 6061 aluminum alloy. High energy electrons impact the plasma chamber wall and sputter the material into the plasma. The alloy has components of magnesium, silicon, titanium, chromium, manganese, iron, copper, and zinc - many of which have been observed background contaminants (see Figure 5).

In order to shield the 6061 aluminum wall from direct exposure to the plasma, the chamber was coated with ultrahigh purity aluminum (99.9995\%). A tungsten coil which had been saturated with the aluminum was suspended in the middle of the plasma chamber. The source was evacuated to $10^{-7}$ Torr and the coil heated resulting in an average surface deposition of 1 micron. Although not all surfaces were adequately coated, namely an injection side disk and mating piece both of which are constructed of 6061 aluminum, the majority of the plasma chamber was coated.

\subsection{Contamination reduction results}

Before the $\mathrm{CO}_{2}$ cleaning, a detailed mass scan of the entire source output was performed with analyzing slits set at $+/-0.1 \mathrm{~mm}$ recording all peaks with an intensity $>1 \mathrm{epA}$. After the $\mathrm{CO}_{2}$ cleaning, the scan was repeated with the exact same source conditions. The argon beam production, which is mainly due to o-ring permeation, was used to monitor the source performance. After the cleaning, the argon output had dropped by $22 \%$ with no shift in the charge state distribution. Reductions in three major contaminants were observed - a factor of 20 reduction for fluorine, a factor of 4 for chlorine, and a factor of 50 for iron.

After the aluminum coating, the mass scan was repeated with the same source settings. The argon beam production had dropped a factor of 3 , mainly in the higher charge states, and our three contaminants were further reduced $-\mathrm{a}$ factor of 160 reduction for fluorine, a factor of 17 reduction for chlorine, and iron was no longer detectable.

While these three components were reduced at the source, the key metric is what is accelerated in the linac and makes it to target. $\mathrm{A}^{98} \mathrm{Zr}$ beam had been produced shortly before aluminum coating the source. After coating, a ${ }^{98} \mathrm{Y}$ beam was produced utilizing the same accelerator tune as the ${ }^{98} \mathrm{Zr}$. Energy spectra were obtained for both beams with the silicon barrier detector as well as with the experimenter's detector. As a result of the coating, several of the stable contaminants observed in the silicon barrier spectrum (Figure 6) had either been eliminated (iron, cadmium, cerium) or had come down significantly (titanium). A significant ${ }^{98}$ Mo peak remained and two new contaminants were introduced $-{ }^{181} \mathrm{Ta}$ and ${ }^{186} \mathrm{~W}$ - presumably due to the tungsten heating coil used for the evaporation which had a $20 \mathrm{ppm}$ component of tantalum and a $10 \mathrm{ppm}$ component of molybdenum. On the experimenter's detector (Figure 7), the ${ }^{98} \mathrm{Mo}$ was reduced by a factor of 5 , the ${ }^{142} \mathrm{Ce}$ was eliminated, but the Ta and $\mathrm{W}$ now dominated the spectrum.

While the above techniques have demonstrated significant reductions in the level of background, especially that due to surface contamination, there are several refinements which can be made. The o-rings need to be eliminated from the source design to establish a truly UHV system. This advancement has been incorporated into the construction of the SPIRAL PHOENIX ECR charge breeder [30]. A new technique to evaporate the aluminum needs to be developed. It is possible that a greater reduction in the ${ }^{98}$ Mo component could have been realized if not for the 10 ppm molybdenum content of the heating coil, as inferred by the significant increase in Ta and W. Several off-line tests with various carbon-based heating elements have been performed with limited success. 


\subsection{Other reduction techniques}

Other ECR charge breeding groups have been pursuing the goal of reduced contamination. The TRIUMF PHOENIX-type ECR breeder initially had a stainless steel plasma chamber which was switched to an aluminum one - realizing a reduction in some of the beam contaminants but not a total elimination. The group then turned to additional stripping of the beam at high energy resulting in a further elimination of contaminants although with a significant penalty in overall efficiency [31].

The group at SPES is pursing clean techniques for the construction and installation of their ECR charge breeder in order to eliminate surface contaminants as well as bulk contaminants. Additionally, they are placing a 1:1000 spectrometer after the ECR breeder. In this way they hope to rid the beam of the majority of contaminants without a reduction in efficiency [32].

\section{EBIS background - ReA-EBIT and REX-EBIS}

For the most part, an EBIS does not have the pervasive background of an ECR ion source due to the lack of interaction with the vessel walls which leads to less foreign species being introduced into the trap region. The EBIS also benefits from a UHV system thus reducing the level of gaseous contaminants. The MSU ReA EBIT, while charge breeding potassium [33], demonstrated a low level of background beam components with a clean enough spectrum to clearly resolve the charge bred potassium beam.

The REX-EBIS group has demonstrated that one will always need to find A/q regions where contaminants are at a minimum, and the team has mapped out their known contaminants based upon the EBIS materials of construction. They can easily choose charge states which avoid these regions resulting in experiments where almost no background beam is making it to target [19].

\section{ANL EBIS performance}

The demonstrated higher efficiencies, shorter breeding times, and greater purity of charge-bred radioactive ion beams achievable with an EBIS have led ANL to develop an EBIS in collaboration with the Brookhaven group [34]. However, the parameters of the electron gun, potential distribution in the ion trap region, electron collector and injection/extraction beam lines are substantially modified from the Brookhaven design in order to obtain the highest acceptance and breeding efficiency of low intensity rare isotope beams. Special attention was paid to the design of the vacuum system to provide high purity of the charge-bred radioactive ion beams. Parameters and some design details of the CARIBU EBIS CB are described elsewhere [35].

The first off-line charge breeding results were obtained in May 2014 [36]. With the injection of a Cs ${ }^{+}$beam, a charge breeding efficiency of $10 \%$ into 14+ was realized with a modest solenoid field $(4 \mathrm{~T})$ and electron beam density (170 $\mathrm{A} / \mathrm{cm}^{2}$ ). There was still a high level of residual background due to several small vacuum leaks which were eventually identified and fixed.

After performing a multi-day bake-out and utilizing a higher magnetic field ( $5 \mathrm{~T})$ and electron beam density (385 $\mathrm{A} / \mathrm{cm}^{2}$ ), the background was substantially lower and we achieved a $20 \%$ breeding efficiency into $28+$ for $28 \mathrm{~ms}$ breeding time, $10^{7}$ ions/pulse without preparation in a cooler/buncher, and a repetition rate of $10 \mathrm{~Hz}$ [21]. For comparison, the best the ECR breeder has achieved is $13 \%$ into $27+$ with a breeding time on the order of $300 \mathrm{~ms}$.

\subsection{EBIS at ATLAS}

The EBIS will replace the ECR charge breeder in late 2015. In addition, the beamline on the CARIBU deck will be reconfigured to incorporate an MR-TOF providing greater mass resolution in the 1:40000 range with $>50 \%$ transmission and a capacity of $10^{4}$ ions/bunch. The system will work at repetition rates between $1-30 \mathrm{~Hz}$. Other than the dipole magnets and a few steerers, all transport elements in the low energy line will be electrostatic. The system is scheduled to be operational in early 2016. 


\subsection{Pulse lengthening schemes}

Unlike the ECR which can continuously accept beam and extract it, the EBIS is inherently a pulsed device. A standard breeding cycle has several parts: the electrostatic barrier is lowered and the 1+ ions enter the trap region, the barrier is raised, the ions are charge bred, and the barrier is lowered releasing the highly charged ions with a typical beam pulse width of 10-20 $\mu$ s. During breeding, new particles cannot enter the trap unless one uses an over the barrier scheme for continuous injection, but this method has thus far been less efficient than pulsed injection [18]. Instead, a cooler/buncher is utilized upstream of the EBIS to accumulate the particles during the breeding cycle and prepare them for injection into the EBIS. The $20 \mu$ s extracted beam pulse width introduces a complication, namely high instantaneous rates on the experimenters' detector.

Various schemes have been developed for lengthening the extracted beam pulse. At REX-EBIS, the trap electrodes and extraction barrier are ramped linearly, producing a beam pulse $>400 \mu$ s in length with an upper limit of $800 \mu \mathrm{s}$ set by the machine acceptance envelope [37].

At MSU for the ReA-EBIT, they have also used the ramping scheme extending the pulse width to $4 \mathrm{~ms}$. In addition, they have worked with a pulse train or comb scheme which produces a quick succession of discrete $2 \mu \mathrm{s}$ pulses, effectively extending beam extraction over a longer time period without additional ramping fields, as shown in Figure 8 [38].

\subsection{Multibeam scheme}

At Argonne, we will utilize the pulse lengthening schemes, but we also see an opportunity to fill the space between beam pulses. With the combination of the new EBIS for radioactive beams and an already existing ECR for stable beams, we can achieve the simultaneous acceleration of two beam species. With the requirement that the A/q be within $1 \%$ of each other, both species are efficiently accelerated in the linac. At the accelerator low energy end, a fast electrostatic deflector will select the ECR or EBIS beam. At the high energy end of the machine, a pulsed kicker directs the beams to the appropriate experimental area. With this scheme, $3 \%$ of the buckets will be filled with radioactive ions and $96 \%$ will be filled with stable ions resulting in a more effective use of machine time.

\section{Ongoing R\&D}

The charge breeding field is rapidly expanding with a lively community pursuing multiple developmental paths in a quest to provide the highest intensity pure radioactive ion beams. The efficiency for ECR sources has been steadily improving and is firmly in the 10-15\% range. This overlaps the performance for $80 \%$ of the beams thus far produced by the existing EBIS charge breeders. The work at ANL on contamination reduction has shown promise with the stable background reduced to a level which allows direct observation of $10^{3}$ pps radioactive beams. The issue of beam contamination with an ECR will be less critical with radioactive beam intensities in the $10^{4}$ to $10^{6}$ regime provided one is operating in a relatively clean region of the spectrum. The pulse lengthening schemes developed by CERN and MSU have increased the extracted pulse length by a factor of 200 partially alleviating concerns of too high instantaneous detector rates. The issue of charge capacity has not yet been directly addressed for an EBIS charge breeder due to the expected secondary beam intensities of most radioactive beam facilities being below the level of concern. As future radioactive beam facilities push the intensity envelope, increased electron beam current and trap size are certain to become active areas of development. Overall, while the issues of beam purity for ECRs and pulse length for EBIS still exist, they are being robustly addressed and the future for both devices appears to be bright.

\section{Acknowledgement}

The author would like to thank J. Alessi, E. Beebe, F. Wenander, P. Delahaye, L. Maunoury, L. Celona, A. Galata, H. Koivisto, O. Tarvainen, T. Lamy, T. Thuiller, A. Lapeierre, T. Baumann, F. Ames, R. Pardo, P. Ostroumov for a great many fruitful discussions, shared results, and collaborative work. This work was supported by the U.S. Department of Energy, Office of Nuclear Physics, under Contract No. DE-AC02-06CH11357. This research used resources of ANL's ATLAS facility, which is a DOE Office of Science User Facility. 


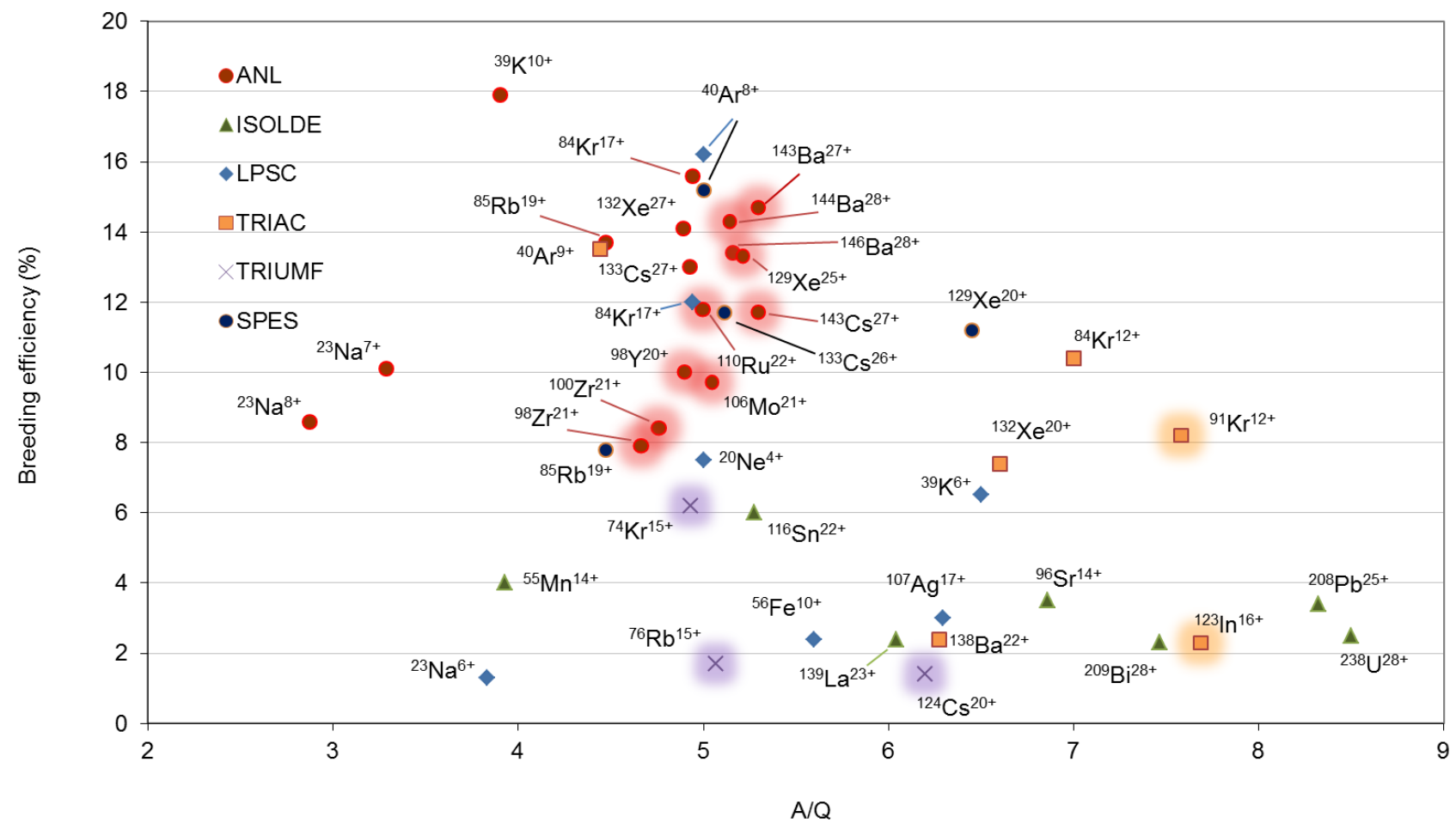

Figure 1: Charge breeding performance for the Phoenix ECR sources tested at ISOLDE and LPSC, the TRIAC source, the TRIUMF source, the ANL source, and the recently commissioned SPES charge breeder (built by LPSC for the LNL group). The radioactive beam species are denoted with a halo around them.

Double column 


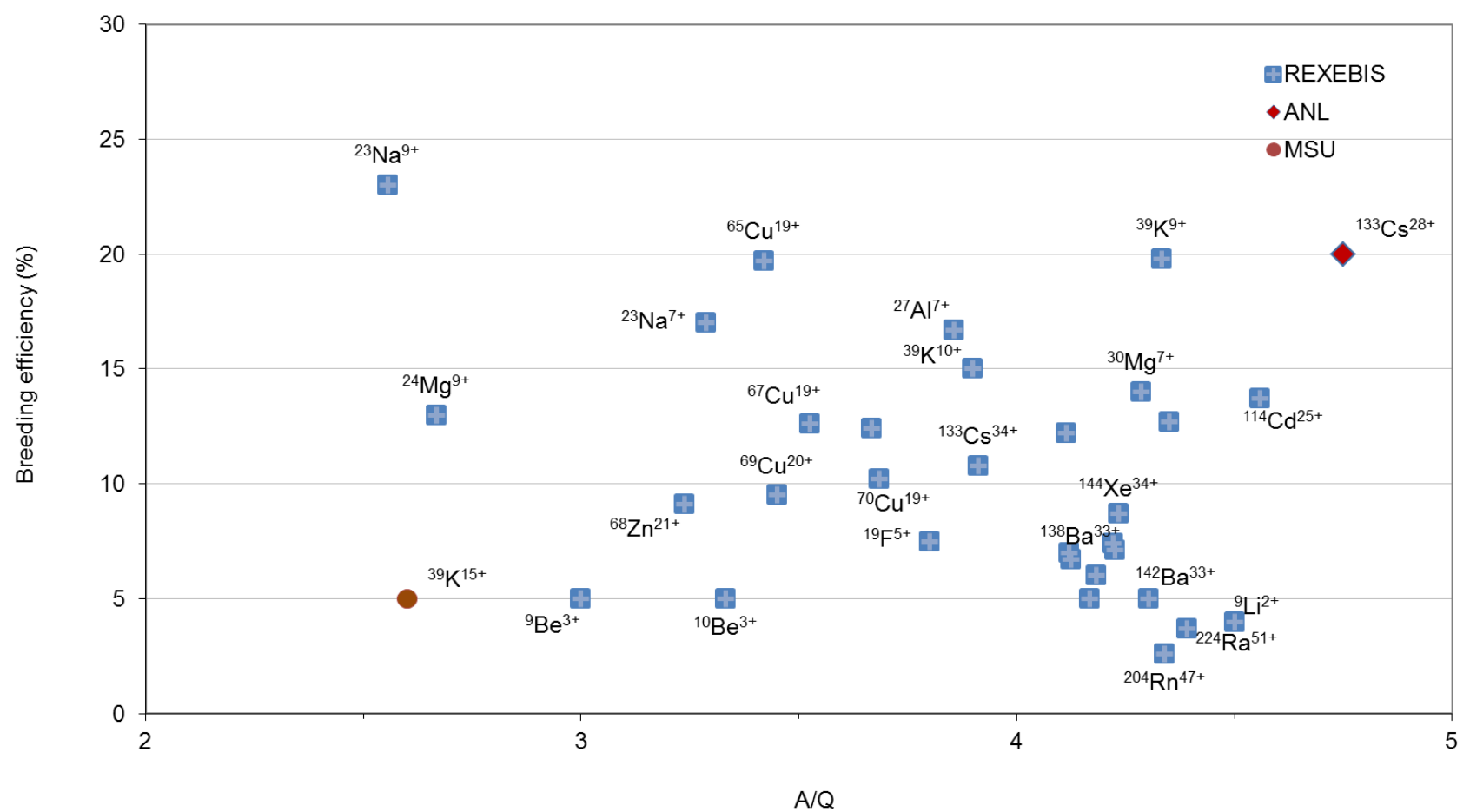

Figure 2: Present EBIS charge breeding performance for the REX-EBIS group, a potassium beam produced by the MSU ReA EBIT in 2013 and a cesium beam recently produced by the ANL EBIS.

Double column 
Figure 3: The Argonne National Laboratory ECR charge breeder. Visible are the two solenoid coils (A), the central plasma chamber which also houses the hexapole (B), the grounded tube (C), and the field shaping iron (D).

Single column 


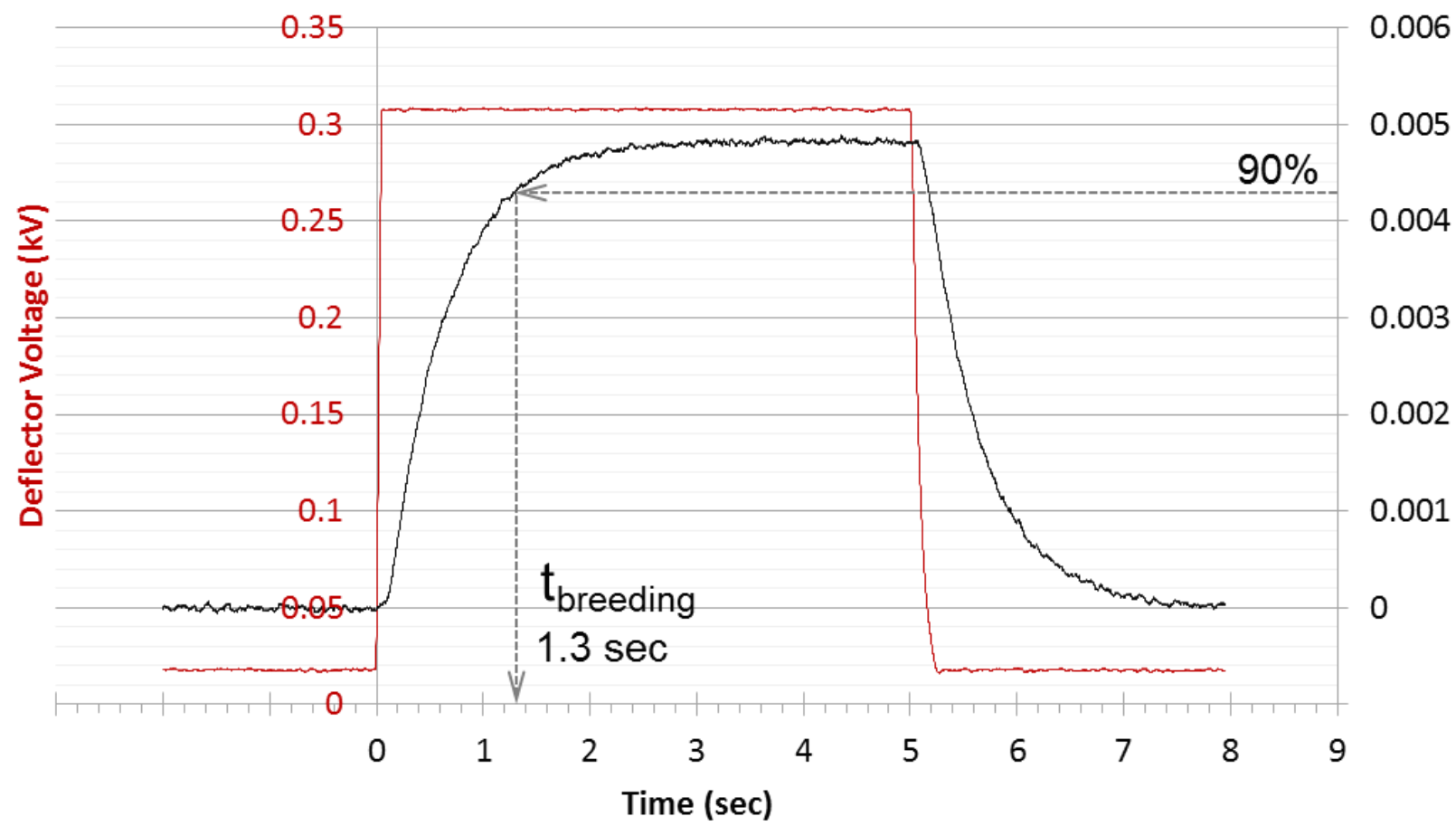

Figure 4: The charge breeding time for ${ }^{132} \mathrm{Xe}^{29+}$ was determined by using an electrostatic steerer (trace shown in red) to pulse the incoming $1+$ beam and measuring the $\mathrm{n}+$ response (trace shown in black). The charge breeding time is defined as the time between injection of the $1+$ beam and the $n+$ beam reaching $90 \%$ of the maximum current.

Single column 
$11.762 \mathrm{GHz}$ operation

$11.765 \mathrm{GHz}$ operation

\begin{tabular}{ccccc}
\hline Charge State & Breeding eff. (\%) & Breeding time $(\mathrm{ms})$ & Breeding eff. $(\%)$ & Breeding time (ms) \\
\hline & & & & \\
$17+$ & 2.3 & 51 & 0.4 & 40 \\
18 & 2.3 & 61 & 0.6 & 91 \\
19 & 3.0 & 75 & 0.8 & 450 \\
20 & 3.7 & 85 & 1.2 & 750 \\
21 & 3.9 & 114 & 1.7 & 950 \\
22 & 5.0 & 103 & 2.7 & 1075 \\
23 & 6.3 & 183 & 4.2 & 1170 \\
24 & 8.9 & 206 & 8.2 & 1150 \\
25 & 10.8 & 230 & 11.0 & 1200 \\
26 & 9.7 & 243 & 13.6 & 1230 \\
27 & 5.6 & & 10.2 & 1366 \\
28 & & & 6.0 & 1350 \\
29 & & & 2.8 & 1330 \\
\hline
\end{tabular}

Table 1: The charge breeding efficiencies and times for all visible charge states of Xe-132. The only change in the ion source operation between the two data sets was a shift in the TWTA operating frequency from $11.762 \mathrm{GHz}$ to $11.765 \mathrm{GHz}$ at a constant $300 \mathrm{~W} \mathrm{RF}$ power.

single column 
Figure 5: Beam of Ba-146 accelerated to $5.4 \mathrm{MeV} / \mathrm{A}$ observed with a silicon barrier detector. A number of stable background components are observed accounting for $97 \%$ of the rate into the detector. Due to there being no strong contaminant near mass 146, the radioactive beam component was still easily identified. Contaminants of titanium, iron, chromium, zinc, molybdenum, tin, and mercury are routinely visible.

Single column 

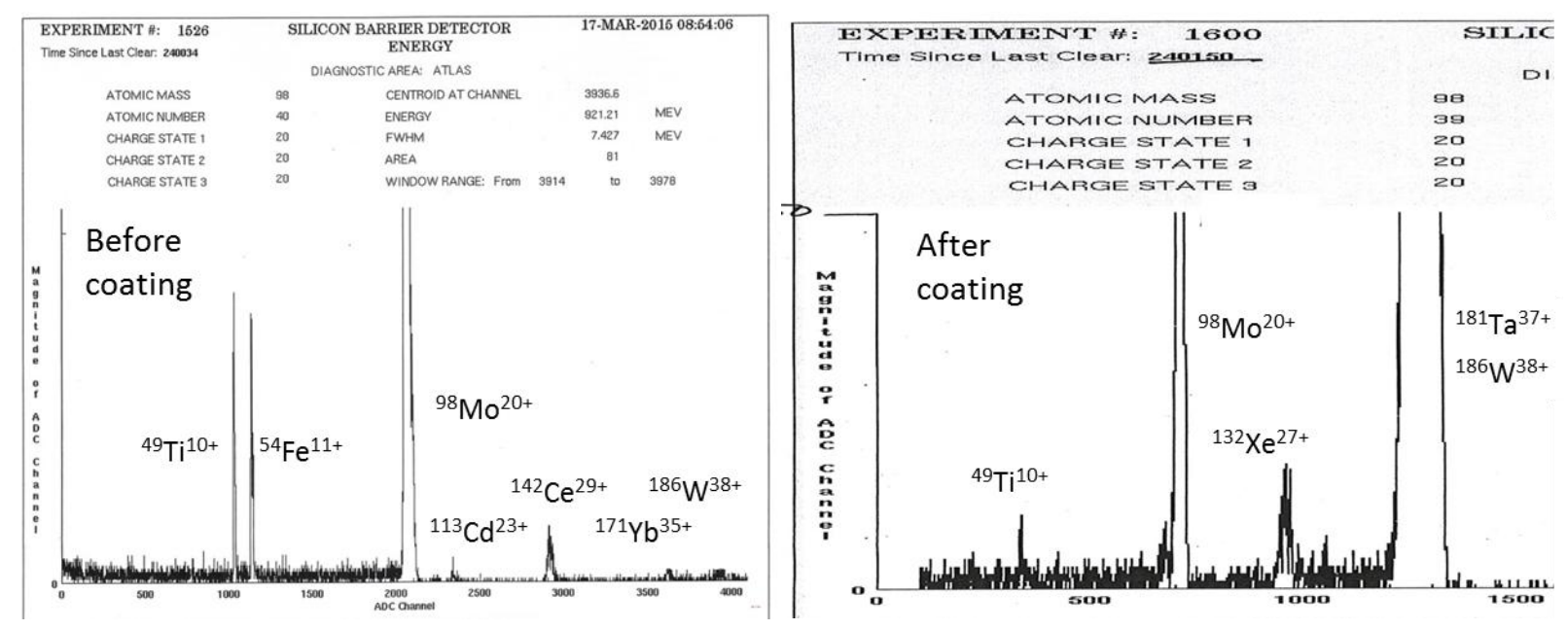

Figure 6: Experimenters' detector for beams of Zr-98 taken before aluminum coating and Y-98 taken after coating. The Mo-98 has been reduced by a factor of 5 and the Ce-142 has been eliminated. New contaminants of Ta and W are now present.

Double column 

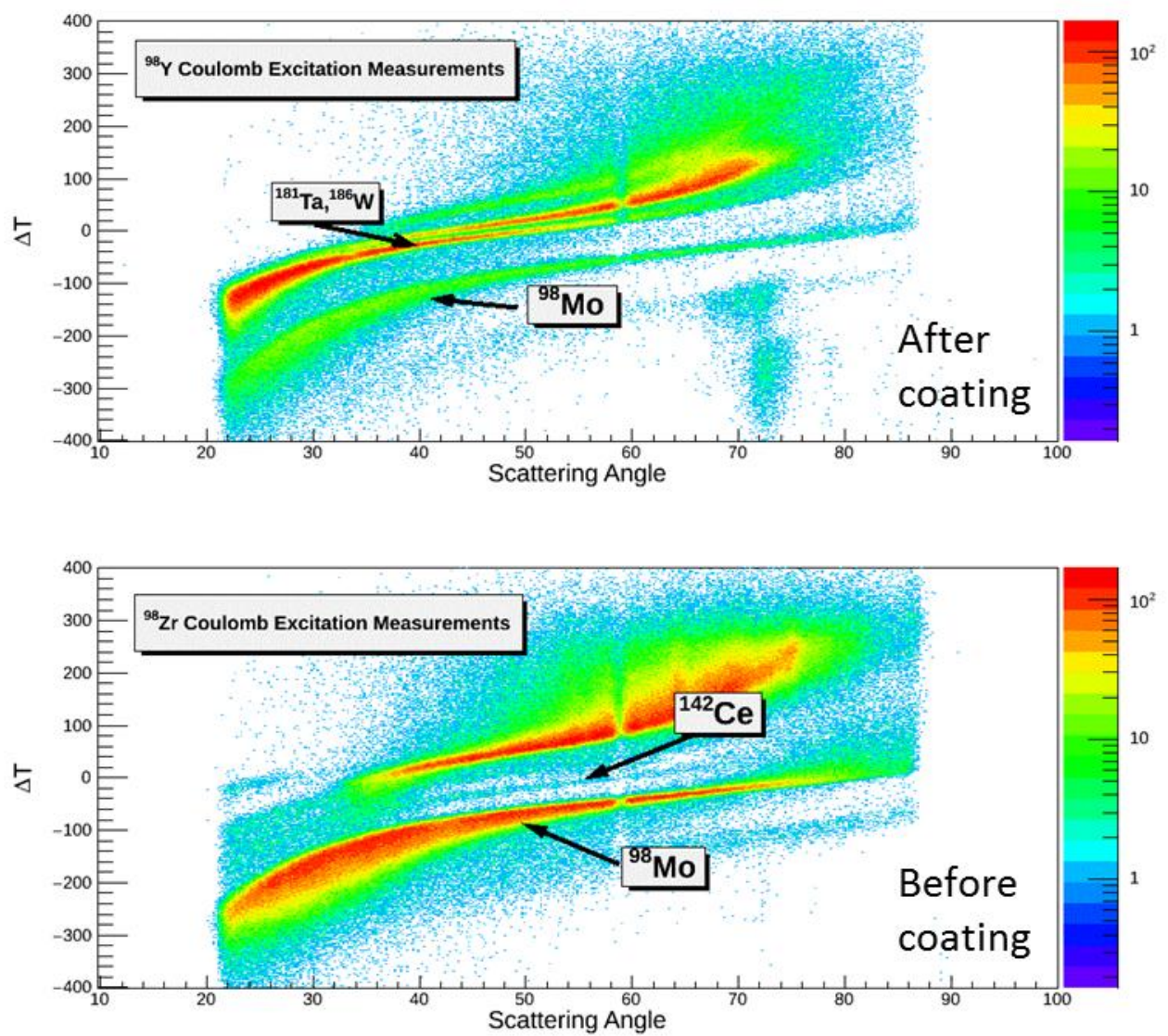

Figure 7: Experimenters' detector for beams of Zr-98 taken before aluminum coating and Y-98 taken after coating. The Mo-98 has been reduced by a factor of 5 and the Ce-142 has been eliminated. New contaminants of Ta and W are now present.

Single column 


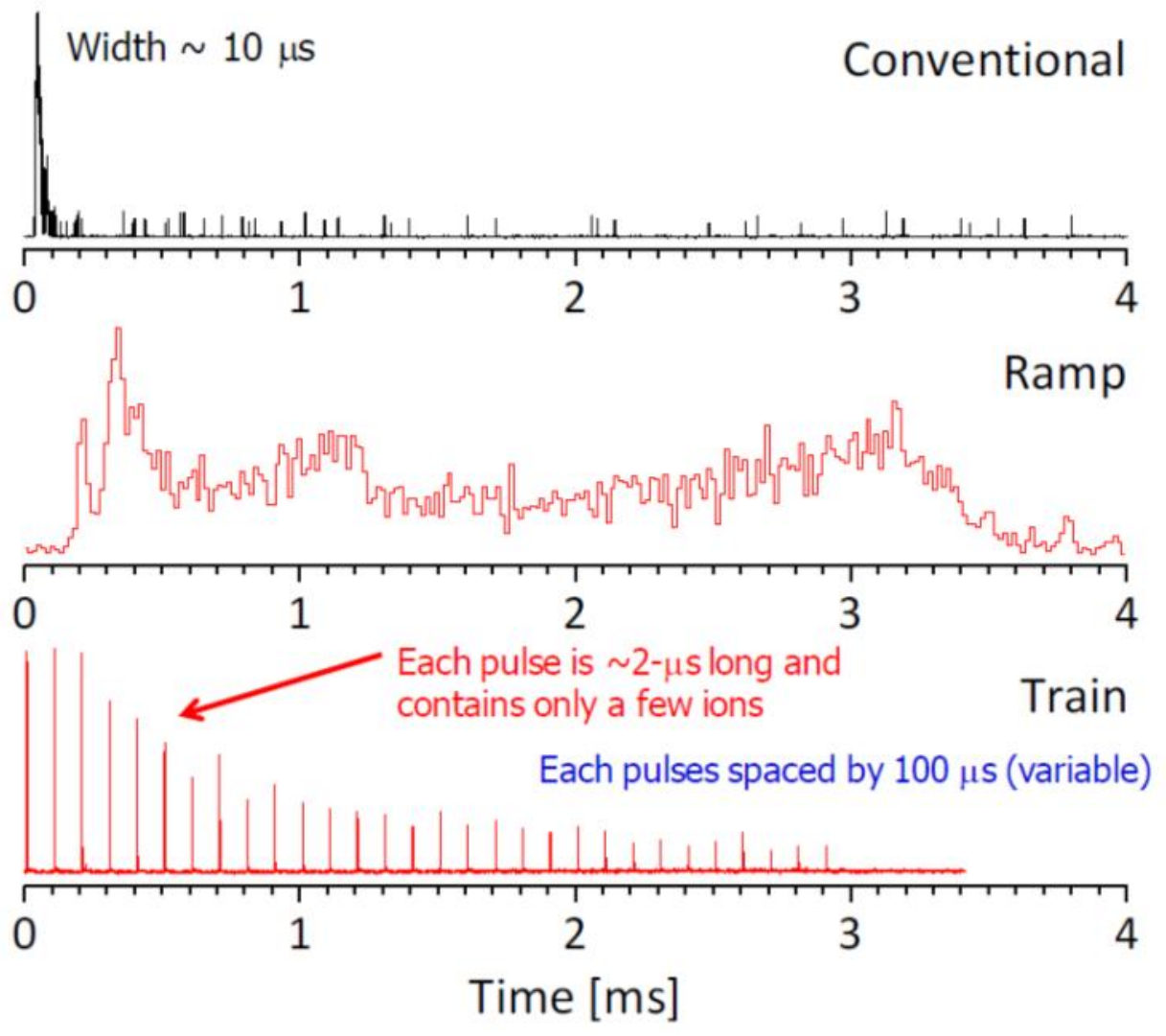

Figure 8: Pulse lengthening schemes utilized at ReA-EBIT at MSU. The conventional method produces a pulse of $10 \mu \mathrm{s}$. For the Ramp scheme, the trap electrodes and barrier potentials are ramped linearly. For the Train scheme the barrier potential is pulsed allowing a sub-set of the charge bred particles to be extracted in $2 \mu$ s packets.

Single column 
[1] G. Prete, A. Andrighetto, L. Biasetto, M. Manzolaro, F. Gramegna, A. Lombardi, A. Pisent, J. Esposito, E. Fagotti, M. Cinausero, P. Mastinu, L. Calabretta, S. collaboration, J. Phys.: Conf. Ser. 168 (2009) 012022

[2] P. Delahaye, L. Maunoury, R. Vondrasek, Nucl. Instrum. Meth. A 693( 2012) p. 104-108

[3] V. Naik, A. Chakrabarti, A. Bandyopadhyay, M. Mondal, S. Dechoudhury, H. Kumar Pandey, D. Bhowmick, D. Sanyal, T. Kundu Roy, J.S. Kainth, T. Kumar Mandi, M. Chakrabarti, P. Karmakar, MOP021, Proc. LINAC08, http://jacow.org/

[4] H. J. Woo, B. H. Kang, K. Tshoo, C. S. Seo, W. Hwang, Y. -H. Park, J. W. Yoon, S. H. Yoo, Y. K. Kim, D. Y. Jang, Journal of the Korean Physical Society, February 2015, Volume 66, Issue 3, pp 443-448

[5] P. Decrock, M. Huyse, G. Reusen, P. Van Duppen, D. Darquennes, Th. Delbar, P. Lipnik, Nucl. Instr. and Meth. B, 70 (1992), p. 182

[6] R. Geller, T. Lamy, and P. Sortais, Rev. Sci. Instrum. 77, 03B107 (2006)

[7] R. Geller, Rev. Sci. Instrum. 71, 612 (2000)

[8] 5T. Lamy, J. L. Bouly, J. C. Curdy, R. Geller, A. Lacoste, P. Sole, P. Sortais, T. Thuillier, J. L. Vieux-Rochaz, K. Jayamanna, et al., Rev. Sci. Instrum. 73, 717 (2002)

[9] P.Delahaye, C. J. Barton, K. Connell, T. Fritio, O. Kester, T. Lamy, M. Lindroos, P. Sortais, G. Transtromer, F.Wenander, Rev. Sci. Instrum. 77, 03B105 (2006)

[10] T. Lamy, R. Geller, P. Sortais, T. Thuillier, Rev. Sci. Instrum. 77, 03B101 (2006)

[11] N. Imai, S. C. Jeong, M. Oyaizu, S. Arai, Y. Fuchi, Y. Hirayama, H. Ishiyama, H. Miyatake, M. H. Tanaka, M. Okada, et al., Rev. Sci. Instrum. 79, 02 A906 (2008)

[12] F. Ames, R. Baartman, P. Bricault, K. Jayamanna, M. McDonald, P. Schmor, T. Spanjers, D. H. L. Yuan, and T. Lamy, Rev. Sci. Instrum. 79, 02A902 (2008)

[13] R. Vondrasek, A. Levand, R. Pardo, G. Savard, R. Scott, Rev. Sci. Instrum. 83, 02A913 (2012)

[14] R. Vondrasek, P. Delahaye, S. Kutsaev, L. Maunoury, Rev. Sci. Instrum. 83, 113303 (2012)

[15] A. Galata, private communication

[16] C. Lyneis, D. Leitner, D. Todd, S. Virostek, T. Loew, A. Heinen and O. Tarvainen, Rev. Sci. Instrum. 77, 03A342 (2006)

[17] R. Becker, Rev. Sci. Instrum. 71 (2000) 816-819

[18] F. Wenander, P. Delahaye, R. Scrivens, R. Savreux, Rev. Sci. Instrum. 77, 03 B104 (2006)

[19] F Wenander 2010 JINST 5 C10004

[20] A. Lapierre, S. Schwarz, K. Kittimanapun, J. Fogleman, S. Krause, S. Nash, R. Rencsok, L. Tobos, G. Perdikakis, M. Portillo, J. A. Rodriguez, W. Wittmer, X. Wu, G. Bollen, D. Leitner, M. Syphers, AIP Conf. Proc. 1525,497 (2013)

[21] P. Ostroumov, A. Barcikowski, C. Dickerson, A. Perry, A. Pikin, S. I. Sharamentov, R. Vondrasek , G. Zinkann, Rev. Sci. Instrum., Accepted for publication

[22] R. Pardo, G. Savard, S. Baker, C. Davids, E. F. Moore, R. Vondrasek, G. Zinkann, NIM-B 261, Issues 1-2 (2007) 965

[23] F. Wenander, Nucl. Instrum. Meth. B266 (2008) p.4346-4353

[24] P. Delahaye, NIM-B, Volume 317, Part B, 15 December 2013, Pages 389-394

[25] R. C. Vondrasek, R. Scott, J. Carr and R. C. Pardo, Rev. Sci. Instrum. 79, 02A901 (2008)

[26] O. Tarvainen, P. Suominen, T. Ropponen, and H. Koivisto, Rev. Sci. Instrum. 77, 03A309 (2006)

[27] L. Celona, G. Ciavola, F. Consoli, S. Gammino, F. Maimone, D. Mascali, P. Spädtke, K. Tinschert, R. Lang, J. Mäder, J. Roßbach, S. Barbarino, R. S. Catalano, Rev. Sci. Instrum. 79, 023305 (2008)

[28] F. Ames, R. Baartman, P. Bricault, K. Jayamanna, Hyperfine Interactions, January 2014, Volume 225, Issue 13, pp 63-67 (10 Oct 2013)

[29] R. Vondrasek, J. Clark, A. Levand, T. Palchan, R. Pardo, G. Savard, R. Scott, Rev. Sci. Instrum. 85, $02 B 903$ (2014)

[30] P. Delahaye, L. Maunoury, R. Vondrasek, Nucl. Instrum. Meth. A 693( 2012) p. 104-108

[31] M. Marchetto, F. Ames, P. Bender, B. Davids, N. Galinski, A. Garnsworthy, G. Hackman, O. Kirsebom, R.E. Laxdal, D. Miller, A.C. Morton, A. Rojas, C. Unsworth, L. Evitts, C. Nobs, FR1A04, Proc. LINAC2012, http://jacow.org/

[32] G. Prete, A. Andrighetto, L. Biasetto, M. Manzolaro, F. Gramegna, A. Lombardi, A. Pisent, J. Esposito, E. Fagotti, M. Cinausero, P. Mastinu, L. Calabretta, S. collaboration, J. Phys.: Conf. Ser. 168 (2009) 012022 [33] D. Leitner, D. Alt, T. M.Baumann, C. Benatti, K. Cooper, B. Durickovich, K. Kittimanapun, S. Krause, A. Lapierre, L. Ling-Ying, F. Montes, D. Morrissey, S. Nash, R. Rencsok, A. J. Rodriguez, C. Sumithrarachchi, S. Schwarz, M. Steiner, M. Syphers, S. Williams, W. Wittmer, X. Wu, FRYBA2, Proc. PAC2013, http://jacow.org/ 
[34] S. Kondrashev, C. Dickerson, A. Levand, P. N. Ostroumov, R. C. Pardo, G. Savard, R. Vondrasek, J. Alessi, E. Beebe, A. Pikin, G. I. Kuznetsov and M. A. Batazova, Rev. Sci. Instrum. 83, 02A902 (2012)

[35] S. Kondrashev, A. Barcikowski, C. Dickerson, R. Fischer, P. N. Ostroumov, R. Vondrasek and A. Pikin, Rev. Sci. Instrum. 85, 02B901 (2014)

[36] S. Kondrashev, A. Barcikowski, C. Dickerson, P. N. Ostroumov, S. Sharamentov, R. Vondrasek and A. Pikin, AIP Conf. Proc. 1640, 54 (2015)

[37] D. Voulot et al., Nucl. Instrum. Meth. B 266 (2008) 4103

[38] T. M. Baumann, A. Lapierre, S. Schwarz, K. Kittimanapun, G. Bollen, AIP Conf. Proc. 1640, 80 (2015); 